Revista Interdisciplinaria de Humanidades, Educación, Ciencia y Tecnología

Año VII. Vol. VII. N². Edición Especial II. 2021

Hecho el depósito de ley: pp201602FA4721

ISSN-L: 2542-3029; ISSN: 2610-802X

Universidad Nacional Experimental Francisco de Miranda (UNEFM). Santa Ana de Coro. Venezuela

Mónica Silvana Jiménez-Ren; Cecilia Ivonne Narváez-Zurita

DOI $10.35381 / \mathrm{cm} . v 7 \mathrm{i} 2.503$

\title{
Control y gestión de costos para la toma de decisiones
}

\section{Control and cost management for decision making}

\author{
Mónica Silvana Jiménez-Ren \\ monica.jimenez.71@est.ucacue.edu.ec \\ Universidad Católica de Cuenca, Cuenca \\ Ecuador \\ https://orcid.org/0000-0002-0652-9090 \\ Cecilia Ivonne Narváez-Zurita \\ inarvaez@ucacue.edu.ec \\ Universidad Católica de Cuenca, Cuenca \\ Ecuador \\ https://orcid.org/0000-0002-7437-9880
}

Recibido: 20 de mayo de 2021

Aprobado: 25 de agosto de 2021 


\title{
RESUMEN
}

La gestión de los costos constituye uno de los elementos más importantes de toda gestión empresarial exitosa, sin embargo, la mayoría de microempresas carecen de esta herramienta, situación que lleva a la toma de decisiones erradas e inclusive pone en riesgo su permanencia. Por tanto, la presente investigación tiene por objetivo estructurar un modelo de planeación del costo de producción para la toma de decisiones acertadas en la Asociación La Vaquerita. Con la aplicación de los instrumentos de investigación, se corroboró que la entidad no planifica la producción y no dispone de una bitácora de anotaciones y registros de los recursos invertidos, por tanto, se deduce que los procesos productivos y administrativos se ejecutan de manera empírica. En consecuencia, se propone un modelo de planeación del costo de producción que incluya un mapeo de producción, pronóstico de demanda, planeación de producción, estructura de costos y punto de equilibrio.

Descriptores: Costes; gestión; planificación; control; toma de decisiones. (Palabras tomadas de Tesauro Unesco).

\begin{abstract}
Cost management is one of the most important elements of all successful business management, however, most micro-enterprises lack this tool, a situation that leads to wrong decisions and even puts their permanence at risk. Therefore, the present research aims to structure a production cost planning model for making the right decisions in the La Vaquerita Association. With the application of the research instruments, it was confirmed that the entity does not plan production and does not have a log of annotations and records of the invested resources, therefore, it is deduced that the productive and administrative processes are executed empirically. Consequently, a production cost planning model is proposed that includes a production mapping, demand forecast, production planning, cost structure and breakeven point.
\end{abstract}

Descriptors: costs; management; planning; control; decision-making. (Words taken from Thesaurus Unesco). 


\section{INTRODUCCIÓN}

Las organizaciones a nivel mundial están en constante evolución, de manera que a lo largo de la historia han experimentado diversos cambios en los ámbitos financiero, económico, político, social, entre otros; por tanto, para enfrentar estos cambios las empresas deben considerar sistemas de información confiables, oportunos y relevantes que aporten en la gestión empresarial con miras a alcanzar mayor competitividad en el mercado actual. Atendiendo a estas consideraciones, la contabilidad de costos constituye la herramienta principal para determinar los costos (Vallejos-Orbe et al. 2019). Dentro de esta perspectiva, toda organización sin importar su tamaño debe interesarse en gestionar de forma adecuada los recursos tangibles e intangibles (elementos del costo) partiendo de la fijación de estrategias para la producción de bienes y servicios, reducción de costos de los recursos productivos y ciclos de fabricación, eliminación de tareas y actividades que no generen valor agregado a la producción, entrega oportuna a los clientes, y en general, estableciendo medidas eficaces para su control (AlcívarCedeño, 2017).

En efecto, en Latinoamérica, uno de los problemas frecuentes que enfrentan las microempresas y que les impide tomar decisiones oportunas y acertadas, es la determinación de los costos. Esto se debe a la inexistencia de procedimientos apropiados y modelos de gestión que posibiliten el registro oportuno, en cuentas contables, de las inversiones que se realizan para la producción de bienes y servicios, situación que pone en riesgo a los negocios e incluso lleva a la toma de decisiones erradas (Álvarez-Morales \& Lazo-Planas, 2017; Bazan-Liñan \& Berrospi-Valverde, 2018).

Con relación a la problemática expuesta, estudios realizados en industrias ecuatorianas del sector lácteo, revelan que la implantación de modelos de gestión y control de costos en algunos casos se han convertido en un gran problema, por cuanto, los procedimientos contables de acumulación establecidos adolecen de errores en la determinación del 
costo, es más, algunas actividades de distribución se sustentan en criterios subjetivos, que de igual manera, mal usados producen imprecisiones en los cálculos (PazquelBarroso, 2015; Alcívar-Cedeño, 2017; Carpio-Valencia, 2019).

La situación descrita, marca un precedente para que las empresas implementen cambios en sus estrategias de costeo que les posibiliten encarar los retos que imponen la competitividad y la globalización, por consiguiente, es fundamental que se analicen los diferentes sistemas de costeo y se determine cuál resulta más apropiado y se adapta a sus necesidades, a fin de evitar fracasos al costear una línea de productos, y por tanto, se optimicen sus procesos y recursos (Albán-Vallejo, Betancourt-Soto, \& MoralesMerchán, 2015; Carpio-Valencia, 2019; Ramos-Farroñan, Mortocarrero-Medina, \& Portocarrero-Medina, 2020). Tan importante es el costeo como primordial es que las pequeñas unidades productivas dispongan de una planeación de producción, que garantice su permanencia y estabilidad a través del tiempo, así como su expansión en el mercado.

A partir de esta argumentación, el presente artículo precisa el estudio de los procesos de producción de la asociación de producción de lácteos la Vaquería Asogualel, dedicada a la producción y comercialización de derivados lácteos, donde se evidencia que el problema radica en la carencia de un sistema de información contable así como un sistema básico de acumulación de costos, lo que imposibilita tener un control apropiado de cada uno de los elementos del costo presentes en las diferentes etapas del proceso productivo para la determinación del precio unitario de cada producto. En este sentido, se fija un precio de venta esporádico con referencia al precio de mercado, estos y otros eventos demuestran que su toma de decisiones obedece principalmente a un elemento empírico, lo cual supone dificultades para la toma de decisiones eficientes.

Con base a lo expuesto, se plantea como problema de investigación: ¿Cómo incide la carencia de gestión y control de los costos para la toma de decisiones en la asociación de producción de lácteos la Vaquería Asogualel, de la provincia de Loja?, y, como 
objetivo general se propone un modelo de planeación del costo de producción que contenga una estructura de costos adaptado a la realidad de la asociación de lácteos la Vaquería Asogualel para la toma de decisiones acertadas por parte de la dirección.

\section{Referencial teórico}

\section{Herramientas de control y gestión de costos}

La mayoría de microempresas surgen sin un plan de negocios, por tanto, no llevan un control y gestión de sus costos, que es esencial para que cualquier empresa sea eficiente, eficaz, productiva, innovadora y orientada hacia la calidad (Mendoza-Torres \& Campos-Mendoza, 2021). Esta situación, marca la necesidad de diseñar, validar e implementar un sistema contable que responda a las características de estas organizaciones, a su vez, permita mantener un control adecuado de sus operaciones a fin de evitar sanciones por la ausencia de registros contables y lo primordial que contribuya a la acertada toma de decisiones.

Dentro de ese marco, para apoyar su desarrollo sustentable, está la contabilidad de costos y administrativa. En este sentido, (Vera-Franco, Espinoza-Cume, \& LópezColoma, 2016) definen a la contabilidad de costos como un "sistema de presentación de información contable que registra, clasifica, asigna, distribuye y controla los costos de las actividades de producción, distribución, administración y financiamiento de una empresa u organización" (p.5). Por otra parte, (Barriga-Pizarro, Asunción-Parralees, SánchezSegarra, \& Balseca-Córdova, 2020) hacen referencia al conjunto de procedimientos y técnicas utilizados para ponderar cuánto cuesta producir un bien o servicio, a la vez, determinar la utilidad o pérdida de aquella producción. En efecto, la contabilidad de costos mide los costos de producción ideando las bases para la planeación, control y toma de decisiones, asegurando así la viabilidad del ente económico (Lazo-Palacios, 2013). 
Respecto a la contabilidad administrativa, (Hansen \& Mowen, 2007) mencionan que se refiere a la forma en que la información contable sobre el control del costo y otra información financiera y no financiera es utilizada para la planeación, el control interno, perfeccionamiento de los procesos operativos y la toma de decisiones eficaces. De este modo, la contabilidad administrativa constituye la base fundamental en la que se apoya la administración, pues, ésta proporciona información relevante encaminada a facilitar las funciones de la gestión empresarial, por lo que, permite a la empresa posicionarse en el mundo empresarial, liderar, ser competitiva y distinguirse de la competencia (Ramírez-Padilla, 2008).

Por consiguiente, se podría decir que tanto la contabilidad de costos como administrativa, constituyen dos herramientas valiosas para el control empresarial, toda vez que, permiten predecir eventos futuros y proporcionan información necesaria y confiable para la toma de decisiones.

\section{Generalidades y sistemas modernos de costos}

El costo es un concepto elemental en la gestión de las organizaciones, así pues, (UribeMarín, 2011) lo define como "todos los rubros en los que se incurre para hacer la transformación de las materias primas con la intervención de los recursos humanos y técnicos, e insumos necesarios para obtener los productos requeridos" (p.4). Por su parte, (Vallejos-Orbe \& Chiliquinga-Jaramillo, 2017) precisa que es "el conjunto de valores incurridos en un período perfectamente identificados con el producto que se fabrica" (p.8). En este sentido, se refiere a los costos generados para la transformación de un bien o servicio, los mismos que comprenden la materia prima (MP), mano de obra (MO) y los costos indirectos de fabricación (CIF) (Arellano-Cepeda, Quispe-Fernández, Ayaviri-Nina, \& Escobar-Mamani, 2017). Bajo esta perspectiva, es importante mostrar los sistemas de costos que pueden implementarse de acuerdo a las necesidades y características de cada organización (ver tabla 1). 


\section{Tabla 1.}

Sistemas de costos.

\begin{tabular}{|c|c|c|}
\hline & características & procedimiento contable \\
\hline $\begin{array}{l}\text { Por órdenes de } \\
\text { producción }\end{array}$ & $\begin{array}{l}\text { Producción esporádica y en pequeñas } \\
\text { cantidades. } \\
\text { Los productos son diferentes y se ajustan a las } \\
\text { descripciones del cliente. } \\
\text { Producción bajo pedido }\end{array}$ & $\begin{array}{l}\text { Se produce a partir de una orden de trabajo. } \\
\text { Por cada orden se abre una hoja de costos que posibilite } \\
\text { determinar el precio unitario del producto. } \\
\text { Cada orden de producción puede contener distinta cantidad de } \\
\text { MP, MO y CIF. }\end{array}$ \\
\hline Por procesos & $\begin{array}{l}\text { Producción continua en grandes cantidades. } \\
\text { Los productos son homogéneos (idénticos). } \\
\text { Producción para existencias }\end{array}$ & $\begin{array}{l}\text { Se da importancia a los costos unitarios, por órdenes } \\
\text { productivos y por período. } \\
\text { Cada unidad productiva, lleva la misma cantidad de MP, MO y } \\
\text { CIF. } \\
\text { Es un sistema apto para aplicar costos estándar. } \\
\text { Origina menor trabajo de oficina. }\end{array}$ \\
\hline $\begin{array}{l}\text { Por actividades } \\
\qquad(A B C)\end{array}$ & $\begin{array}{l}\text { Es una herramienta clave para el aumento de } \\
\text { la competitividad. } \\
\text { Los CIF se fijan y distribuyen con relación a } \\
\text { cada actividad }\end{array}$ & $\begin{array}{l}\text { El costo de producción resulta de la suma de todos los costos } \\
\text { incurridos en cada actividad. } \\
\text { Los costes se pasan a las actividades en una primera fase y } \\
\text { posteriormente desde éstas se llevan a los productos }\end{array}$ \\
\hline
\end{tabular}

Adaptado de: Vinza-Romero (2012).

De los sistemas de costos revisados, el costeo por órdenes de producción es aplicable a la microempresa Asogualel, pues, la producción es periódica, esporádica, en lotes y en pequeñas cantidades; por tanto, se presenta un enfoque más detallado de este sistema: Sistema de costos por órdenes de producción. Se caracteriza en general porque permite: a) a través de la hoja de costos llevar un registro íntegro y sistemático de los tres elementos del costo, para el cálculo del costo de producción real de cada orden; b) conocer el proceso de producción para dar seguimiento en cada etapa de fabricación; c) controlar de forma constante la producción que permita en lo posible la reducción de costos en producciones futuras.

Bajo este sistema, cuando se recepta un pedido, de inmediato se emitirá una orden de producción o de trabajo, a la par se debe abrir una hoja de costos, donde se irá acumulando de manera sistémica todo cuanto se invierte en la fabricación del producto y deberá actualizarse conforme se utilicen y apliquen a los elementos del costo, finalizada la producción se la liquida (Zapata-Sánchez, 2019). 
A continuación, se hace énfasis en los tres elementos primordiales que intervienen en todo proceso productivo: materiales o MP, fuerza laboral (MO) y otros insumos (CIF): Materiales (MP): El autor (Sarmiento-Regalado, 2005) define a los materiales como "un elemento principal del costo, siendo este en muchos casos el más importante para la transformación en el artículo terminado, su principal característica es la fácil identificación en cantidad, peso y volumen" (p.13). Se subdivide en: materia prima directa (MPD) que conforma la parte integral del bien en producción y se puede identificar en la fabricación, en contraste; materia prima indirecta (MPI) forma parte de la elaboración de un producto, no se puede determinar con precisión en la producción. Por su parte, (ZapataSánchez, 2019) señala que, a través de siete fases se desarrolla el ciclo de este elemento: a) determinación de necesidades; b) cotización y selección del proveedor; c) emisión de la orden de compra; d) recepción y verificación de pedido; e) acondicionamiento y despacho; f) devoluciones internas y externas; g) pago a proveedores.

Fuerza laboral (MO): Denominado también trabajo fabril, representa el componente humano de la producción, sin cuya intervención no se podría realizar la actividad fabril (Garrison, Noreen, \& Brewer, 2007). Cuando su identificación es compleja en la producción, se trata como CIF. En este rubro, las horas improductivas normales (ensamblaje y desmontaje de materiales) se cargan al costo de las órdenes, por el contrario, las horas improductivas por acontecimientos que pueden evitarse $o$ al menos reducirse se cargan a los resultados (Zapata-Sánchez, 2019).

Con base en lo expuesto, la administración de talento humano, es esencial en la fabricación de bienes, por lo que, es necesario reclutar a las personas más idóneas, adiestrarlas, a su vez, capacitarlas y evaluarlas con frecuencia para su promoción y reclasificación. Así mismo, se deben implementar controles básicos y métodos enfocados a la separación de funciones y actividades que se desarrollan dentro de las organizaciones (Zapata-Sánchez, 2019). En esencia, su administración y control 
adecuado determina e influye de manera significativa en el costo definitivo del producto y/o servicio.

Otros insumos (CIF): en la actualidad, Los CF tienen un valor representativo en la estructura general del costo total, en efecto, algunas actividades y tareas que realizaba la clase obrera están siendo desarrolladas por máquinas, en consecuencia, se han incrementado los servicios básicos, servicios de mantenimiento y seguros. La depreciación es significativa, la necesidad de combustible es mayor, etc., por tal razón, no forman parte del producto final y se dividen en MPI, mano de obra indirecta (MOI) y otros CIF (Morocho-Malla, Narváez-Zurita, \& Erazo-Álvarez, 2019).

Debido a las características de los CIF, resulta complejo asignarlos a las hojas considerando los costos históricos o reales, por tanto, se debe preparar el presupuesto que da origen a la tasa predeterminada (TP). Una forma conveniente, aunque imprecisa consiste en presupuestar los CIF, a partir de éstos, se obtiene una tasa o alícuota que debe registrarse en la hoja de costos, acorde a la orden de producción y en secuencia como se vaya finiquitando cada pedido (Zapata-Sánchez, 2019).

\section{Sostenibilidad y crecimiento de pequeñas unidades productivas}

La globalización y las nuevas tecnologías ponen a prueba a la industria, por su parte, el sector lácteo de América Latina viene encarando un sinnúmero de desafíos, lo que implica que, se piense en acciones y estrategias encaminadas al perfeccionamiento de las unidades asociativas que parten de un trabajo integral, coordinado y consensuado de los agentes del territorio, esto permitirá de a poco ir fortaleciendo su estructura y conseguir un posicionamiento local como base para su expansión en nuevos mercados (Rodríguez-Espinosa, Ramírez-Gómez, \& Restrepo-Betancur, 2017).

Al mismo tiempo, se debe enfocar hacia el mejoramiento socio-organizativo, sin desatender el ámbito empresarial, mediante el diseño de un plan de producción que permita optimizar los recursos de la empresa sacando el mayor provecho. De manera 
que, la entidad tenga definido la cantidad a producir y el tiempo que conlleva hacerlo. Como complemento, es preciso, hacer proyecciones con base en el comportamiento de la oferta, la demanda y la competencia, de igual manera, resulta práctico determinar el punto de equilibrio a fin de estimar bajo qué circunstancias se podría superar esta base, para producir con la rentabilidad deseada, siendo imprescindible tomar como plataforma un plan de producción, proyección de ventas, requisiciones y ganancias esperadas. Aquello, se puede alcanzar mediante una gestión acertada de los costos (RamírezMolinares, García-Barbosa, \& Panoja-Algarín, 2010).

Sobre el asunto, es preciso hacer referencia al precio de venta, el cual se establece tomando en consideración los costos y gastos totales con relación a las unidades producidas añadiendo un margen de ganancia (Albán-Vallejo, Betancourt-Soto, \& Morales-Merchán, 2015). No obstante, previo a tomar una decisión empresarial, se debe considerar: a) el estudio de mercado para valorar la acogida del producto; b) plan de marketing que incluya estrategias de promoción y distribución; c) análisis de la curva de la demanda para la fijación del precio de venta (curva de la demanda); d) calcular la inversión en la producción del servicio o producto; e) considerar la normativa que rige al ente económico y peculiaridades de la competencia; f) determinar la base para la fijación de precios (encarar a la oposición, incrementar las ganancias, lograr estabilidad económica) (Condori-Muñiz \& Maraza-Humpire, 2017).

De este modo, la gestión empresarial busca incrementar la rentabilidad, sin descuidar el fortalecimiento y crecimiento empresarial, adicionar valor y demás aspectos que le permitan progresar.

\section{Toma de decisiones estratégicas en las organizaciones}

La toma de decisiones es un proceso continuo que realizan los gestores empresariales, dado que, la supervivencia de las organizaciones actuales depende cada vez más de las decisiones que se toman. Por tanto, supone la elección de la mejor alternativa de entre 
las existentes para confrontar y dar solución al problema encontrado. Sin embargo, la toma de decisiones en microempresas, en la mayoría de los casos se hace de forma empírica, sin previa planificación, es más, no se analiza a fondo el problema, sus causas y efectos. para de entre posibles soluciones elegir la mejor estrategia (FranklinFincowsky, 2011; Pilay-Toala, 2015; Artieda, 2015).

No obstante, (Zapata-Sánchez, 2019) sugiere el presente proceso, para que, en parte la decisión electa tenga los efectos esperados: 1) identificar el problema o la situación actual; 2) revisar las causas que condujeron a la situación o problema encontrado relacionado con los costos y logros futuros; 3) con base a ciertos supuestos, realizar proyecciones sobre costos y beneficios esperados; 4) elegir una opción, de entre las propuestas y que resulte ser la más viable; 5) poner en práctica la opción escogida; 6) evaluar el desempeño de la decisión.

En correspondencia con la información que antecede, se debe precisar que una toma de decisión inteligente y estratégica es sumamente trascendental en toda organización, pues, involucra el análisis de datos y estudio del entorno, en efecto, se puede afirmar que existen los siguientes tipos de decisiones: a) decisiones estratégicas: se orientan al logro de planes a largo plazo; b) administrativas: aplicables en el mediano plazo, pues, buscan optimizar la gestión de los medios y recursos para el desempeño empresarial; c) corrientes: su ejecución por lo general es inmediata (corto plazo); d) estructuradas: obedece a ciertas fases de implementación; e) no estructuradas: son decisiones imprevisibles, por tanto, no tienen una estructura definida (Cortés-Cortés, 2015).

Retomando la idea inicial, una de las principales funciones de los directivos es la planificación, entendida como el proceso de toma de decisiones para alcanzar los objetivos previamente trazados. En este caso, es esencial que las organizaciones se interesen en desarrollar prácticas de gestión de costos innovadoras y relevantes, y a su vez, que la administración ejecute estas actividades primordiales: planeación, dirección y control (ver figura 1). 


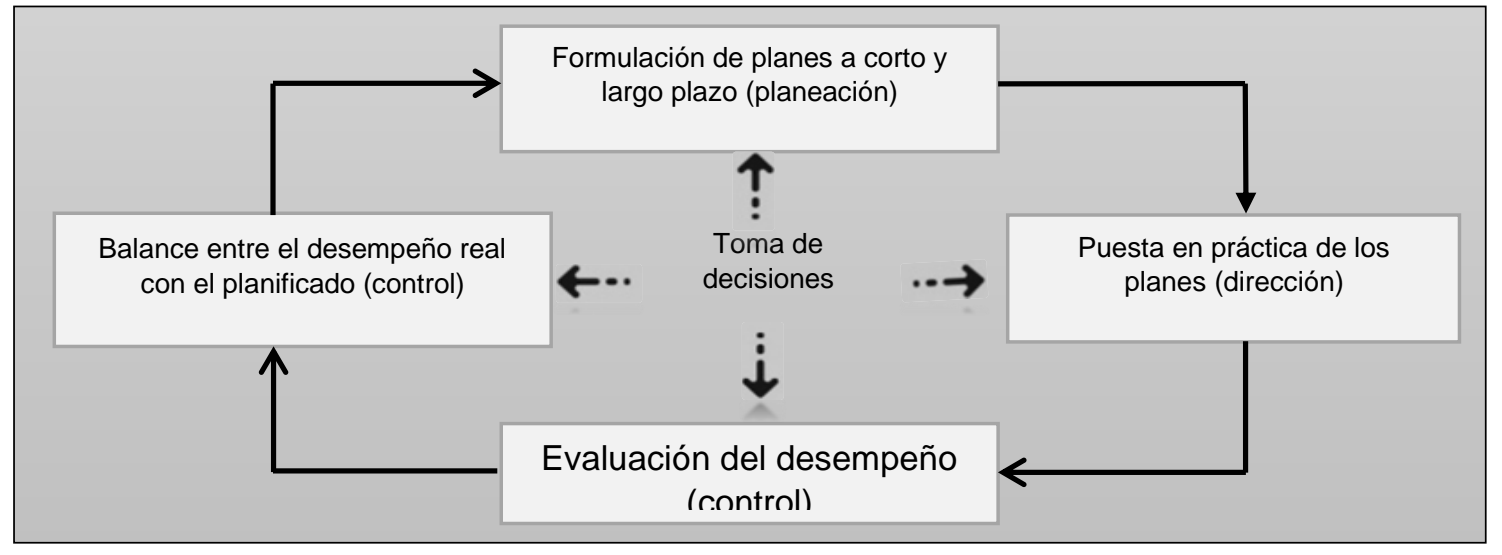

Figura 1. Proceso de planeación y control.

Fuente: Garrison, Noreen, \& Brewer (2007).

Los temas tratados en el presente artículo ponen de manifiesto la importancia de implementar un plan estratégico en cualquier entidad, independientemente del tipo que sea y del sector al que pertenezca, donde deberán reflejarse las estrategias propuestas y las acciones para lograrlas, proponiendo alternativas que generen efectos cambiarios positivos, considerando la mejora continua para satisfacer necesidades de los clientes y asociadas a los factores internos y externos que afecten a la propia empresa. Como complemento, es preciso disponer de información confiable y oportuna que facilite el proceso, pues, de la calidad de la información depende la toma de decisiones organizacionales y por consiguiente repercute en la efectividad de la estrategia propuesta. 


\section{METODOGÍA}

La investigación se enmarcó en un diseño no experimental, de tipo descriptiva de manera que, en la parte inicial se describieron las nociones y teorías de las variables de estudio y en la fase de la propuesta se detalla la medición, valoración y registro de los elementos del costo respecto de la unidad de análisis. Para este estudio, se levantó información en un solo período de tiempo (finalidad transversal).

En el caso del cuestionario, fue sometido a un proceso de validación de contenido a partir del juicio de expertos, con los resultados obtenidos se perpetró el cálculo del índice de validez de contenido (IVC), alcanzando un coeficiente de 0.94 , lo que demuestra que cada ítem está dentro del rango de aceptabilidad, por tanto, es congruente y útil para la medición de los conceptos susceptibles de evaluación. El universo de estudio, estuvo integrado por 10 personas entre socios y directivos de la asociación de producción de lácteos la Vaquería Asogualel, del cantón Loja.

\section{RESULTADOS}

Con el propósito de identificar la situación operativa y funcional de Asogualel, así como, los problemas potenciales relacionados con la planeación y control del costo de producción para la correcta toma de decisiones, se proceden a aplicar encuestas a los socios y entrevistas a los directivos. Del análisis efectuado, se desprenden los siguientes resultados relevantes:

Identificación y análisis de problemas para la toma de decisiones: uno de los aspectos más significativos en el sector empresarial, es la toma de decisiones, las cuales, resultan determinantes para garantizar el éxito de la entidad. Por tanto, es importante contar con herramientas que faciliten esta compleja tarea. Sin embargo, se constató que la entidad estudiada carece de herramientas para la planeación y la toma de decisiones. En efecto, el $100 \%$ de los encuestados señalan que las decisiones no son 
planificadas ni obedecen a un procedimiento lógico, lo cual inicie con la valoración y análisis del problema para definir la meta deseada (ver figura 2).

Criterios de decisión: entre los cimientos principales en los que se fundamenta la toma de decisiones está la información que se disponga. A pesar de esto, se ratificó que el ente económico no dispone de estados financieros que permitan optimizar la toma de decisiones, en consecuencia, cuando existe un inconveniente en la organización, a breves rasgos se identifica el problema sin analizar a fondo sus causas, efectos y riesgo de tomar una decisión, así como el costo de su implementación (ver figura 2).

Puesta en práctica de la estrategia: aunque no se ha definido una fórmula que señale el momento exacto para implementar una estrategia, existen buenas prácticas que conducen a la toma de decisiones exitosas. No obstante, se constató que, no se analiza con precaución una estrategia de solución o alternativa para de entre varias opciones seleccionar e implementar la más idónea. Por tanto, se expone la situación, se discuten las alternativas, se escoge por mayoría de votos y se llega a definir la acción a tomar y su estrategia de implementación (ver figura 2).

Evaluación de las decisiones: una vez tomada una decisión e implementada la estrategia, es muy importante dar seguimiento a la misma con el propósito de detectar posibles imprevistos y actuar con antelación. Empero, el 100\% de los encuestados aseveran que, no se da seguimiento a los resultados obtenidos para actuar ante futuras situaciones similares, llama la atención que, no se han definido indicadores que permitan verificar si la estrategia tomada generó efectos positivos o, por el contrario, se deben aplicar nuevas medidas (ver figura 2). 


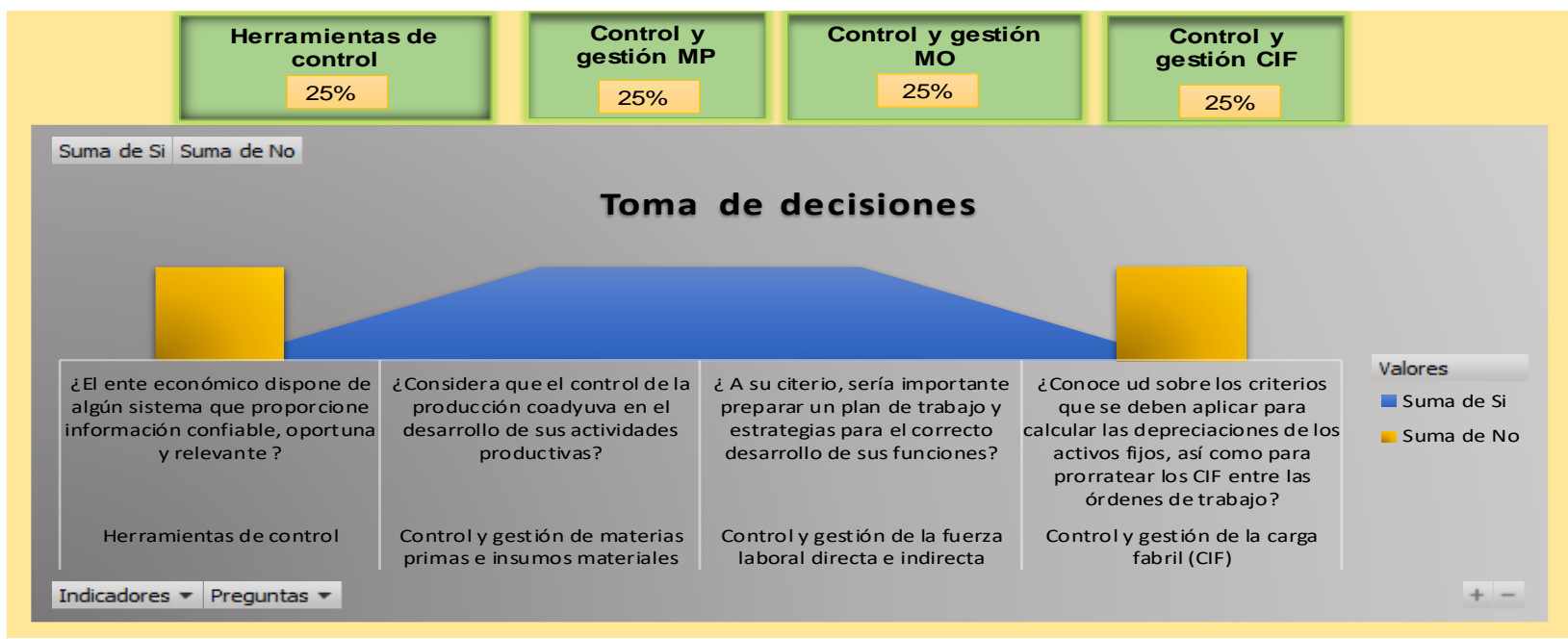

Figura 2. Resultados encuesta referente a la variable costo de producción. Elaboración: Los autores.

Herramientas de control: a pesar de que esta microempresa está regulada por la Superintendencia de Economía Popular y Solidaria (SEPS), que le obliga a presentar información confiable, oportuna y relevante que se obtiene de la contabilidad bajo normas internacionales, sus asociados ratifican que Asogualel, solo mantiene un libro de ingresos y gastos rudimentario, incompleto y poco confiable (ver figura 3), de manera que, si no se corrige a tiempo esta situación, puede ser objeto de sanciones futuras. Incluso, se observó que la organización no dispone de un sistema de acumulación de costos para el control y seguimiento de MP, MO y CIF que se utilizan en cada lote de producción.

Control y gestión de materias primas e insumos materiales: los directivos de la empresa están conscientes de que el control de la producción, es quizá, la parte más importante del desarrollo de sus actividades (ver figura 3). Sin embargo, se observó que, para iniciar el proceso productivo, no se expide la orden de trabajo ni se emite la respectiva hoja de costos, mucho menos, se elaboran notas de despacho de materiales 
y otros elementos embodegables. Por otro lado, no se dispone de un plan de compras en el que se identifique con certeza la cantidad periódica (anual o mensual) necesaria y las fechas tentativas de adquisición.

Control y gestión de la fuerza laboral directa e indirecta: la gestión de la fuerza laboral es una tarea compleja que define el futuro empresarial. Bajo esta perspectiva, el $100 \%$ de encuestados coindicen en que, se debe preparar un plan de trabajo y estrategias para el correcto desarrollo de sus funciones (ver figura 3). Aun así, la entidad no cuenta con un organigrama funcional en el que se describa de forma clara y precisa las funciones que debe cumplir cada departamento o unidad. Así mismo, se puso en evidencia que la MO no se controla mediante una tarjeta individual de asistencia ni se dispone de mecanismos que aseguren la presencia, permanencia y cumplimiento de tiempos en la ejecución de las tareas y objetivos.

Control y gestión de la carga fabril (CIF): de los tres elementos del costo de producción, el más complejo es la carga fabril, en el sentido que, no se pueden asignar de manera exacta a una sola unidad de producto, por tanto, se distribuyen mediante el uso de inductores. De lo expuesto, se desprende que, en la asignación de costos no se incluyen todas aquellas erogaciones en las que incurre la entidad para la producción. Como resultado se obtuvo que en su totalidad desconocen los criterios para calcular las depreciaciones de los activos fijos, así como para prorratear los CIF entre las órdenes de trabajo (ver figura 3). 
Revista Interdisciplinaria de Humanidades, Educación, Ciencia y Tecnología

Año VII. Vol. VII. N². Edición Especial II. 2021

Hecho el depósito de ley: pp201602FA4721

ISSN-L: 2542-3029; ISSN: 2610-802X

Universidad Nacional Experimental Francisco de Miranda (UNEFM). Santa Ana de Coro. Venezuela

Mónica Silvana Jiménez-Ren; Cecilia Ivonne Narváez-Zurita

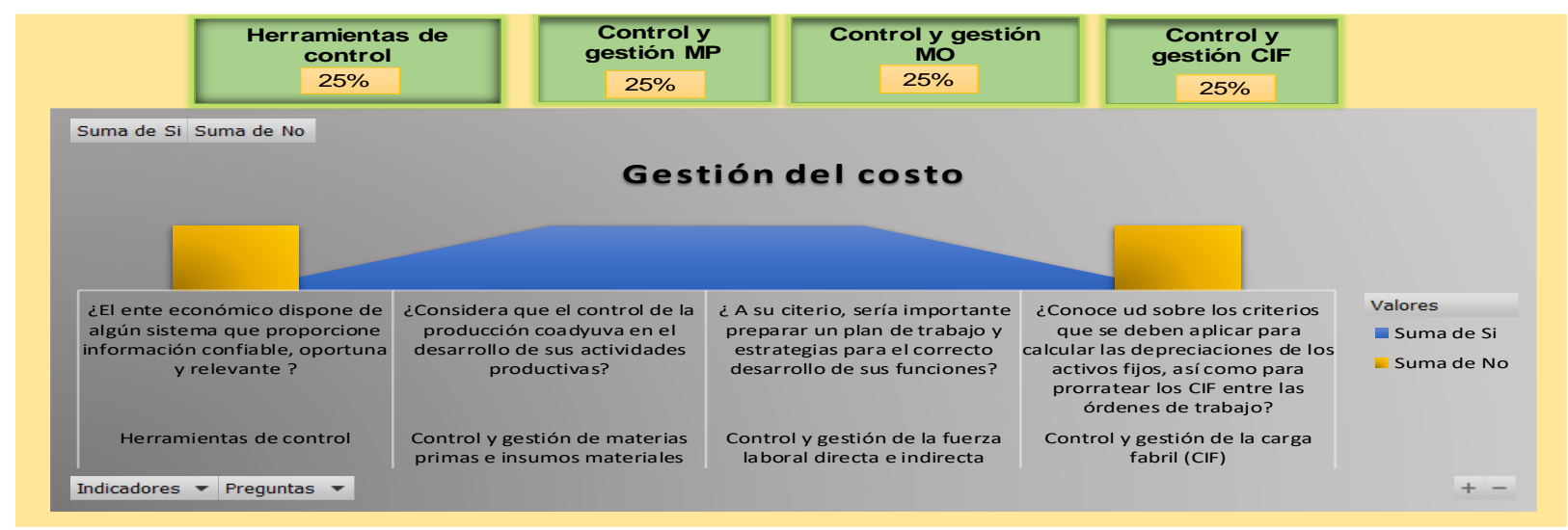

Figura 3. Resultados encuesta referente a la gestión del costo.

Elaboración: Los autores.

\section{PROPUESTA}

Considerando que Asogualel, según los resultados del diagnóstico, carece de herramientas para la planeación y control de los costos de producción, lo cual impide la optimización de sus recursos, se propone a continuación un modelo de planeación del costo de producción (ver figura 4) que contiene una estructura de costos adaptado a la realidad de la empresa con el fin de mejorar la toma de decisiones acertadas por parte de la dirección. 


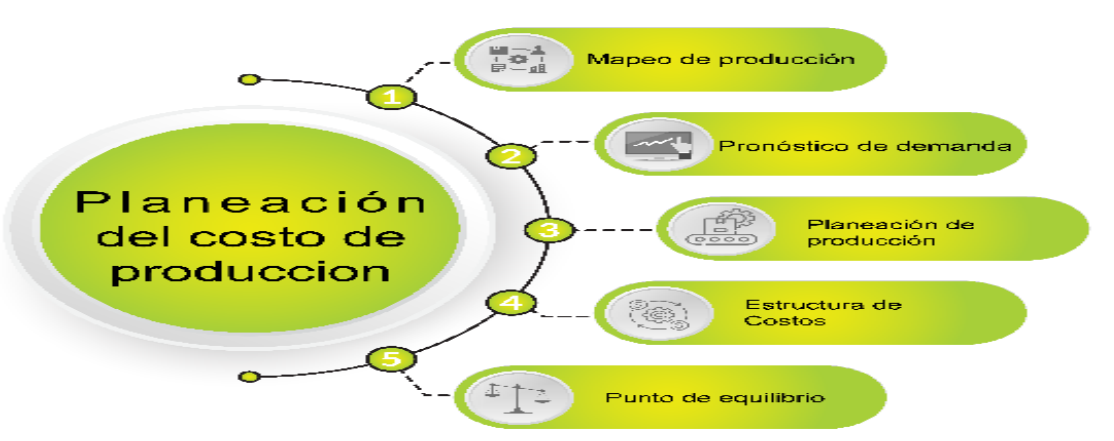

Figura 4. Planeación del costo de producción. Elaboración: Los autores.

1. Mapeo de producción: luego de observar las operaciones de fabricación de los derivados lácteos, en la figura 5, se muestran el diseño esquemático del flujo operacional de producción de quesos, por ser el producto más solicitado y con alto impacto en las ventas.

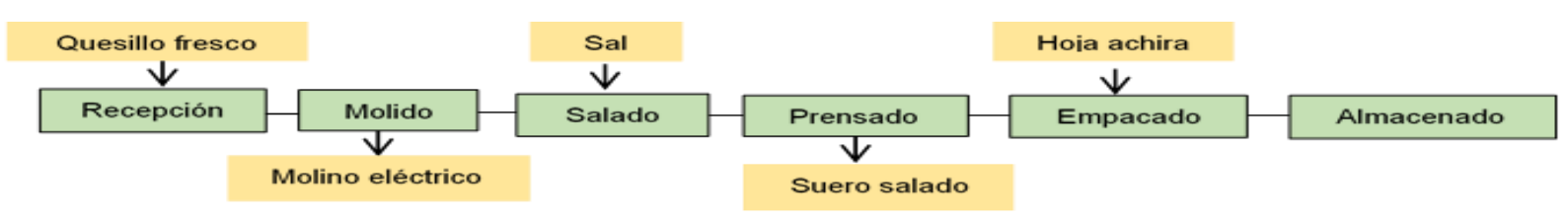

Figura 5. Flujograma elaboración del queso.

Elaboración: Los autores.

2. Pronóstico de demanda: con la finalidad de estimar la potencial demanda de los derivados lácteos para el año 2022, se emplea el modelo de regresión lineal simple, a partir de los despachos derivados de los meses de enero 2020 a mayo 2021. En la figura 6 se ejemplifica el pronóstico de demanda de quesos, los 
Revista Interdisciplinaria de Humanidades, Educación, Ciencia y Tecnología

Año VII. Vol. VII. N². Edición Especial II. 2021

Hecho el depósito de ley: pp201602FA4721

ISSN-L: 2542-3029; ISSN: 2610-802X

Universidad Nacional Experimental Francisco de Miranda (UNEFM). Santa Ana de Coro. Venezuela

Mónica Silvana Jiménez-Ren; Cecilia Ivonne Narváez-Zurita

resultados, se usan como referencia para que este centro de distribución tenga el abasto óptimo y se evite exceso de stock.

\begin{tabular}{|c|c|c|c|c|c|c|c|c|c|}
\hline & & $3059=$ & Pror & o de la & emanda & ogualel & & & \\
\hline \multicolumn{10}{|c|}{ Producto: queso maduro en hoja de achira } \\
\hline Variables & $\mathbf{x}$ & $\mathbf{Y}$ & $\mathbf{X} \times \mathbf{Y}$ & $x^{2}$ & \multicolumn{3}{|c|}{ Año 2022} & \multirow{2}{*}{ valor unitario } & \multirow{2}{*}{ Total } \\
\hline meses & periodo & demanda & & & meses & periodo & Proyección & & \\
\hline ene-20 & 1 & 180 & 180 & 1 & ene-22 & 18 & 208 & 2.00 & $\$ \quad 415.00$ \\
\hline feb-20 & 2 & 180 & 360 & 4 & feb-22 & 19 & 209 & 2.00 & $\$ \quad 418.24$ \\
\hline mar-20 & 3 & 180 & 540 & 9 & mar-22 & 20 & 211 & 2.00 & $\$ \quad 421.47$ \\
\hline abr-20 & 4 & 180 & 720 & 16 & abr-22 & 21 & 212 & 2.00 & $\$ \quad 424.71$ \\
\hline may-20 & 5 & 180 & 900 & 25 & may-22 & 22 & 214 & 2.00 & $\$ \quad 427.94$ \\
\hline jun-20 & 6 & 180 & 1080 & 36 & jun-22 & 23 & 216 & 2.00 & $\$ \quad 431.18$ \\
\hline jul-20 & 7 & 200 & 1400 & 49 & jul-22 & 24 & 217 & 2.00 & $\$ \quad 434.41$ \\
\hline ago-20 & 8 & 200 & 1600 & 64 & ago-22 & 25 & 219 & 2.00 & $\$ \quad 437.65$ \\
\hline sep-20 & 9 & 200 & 1800 & 81 & sep-22 & 26 & 220 & 2.00 & $\$ \quad 440.88$ \\
\hline oct-20 & 10 & 200 & 2000 & 100 & oct-22 & 27 & 222 & 2.00 & $\$ \quad 444.12$ \\
\hline nov-20 & 11 & 200 & 2200 & 121 & nov-22 & 28 & 224 & 2.00 & $\$ \quad 447.35$ \\
\hline dic-20 & 12 & 200 & 2400 & 144 & dic-22 & 29 & 225 & 2.00 & $\$ \quad 450.59$ \\
\hline ene-21 & 13 & 200 & 2600 & 169 & \multirow{2}{*}{\multicolumn{3}{|c|}{$\mathrm{b}=\frac{N \sum x y-\sum x \sum y}{N \sum x^{2}-\left(\sum x\right)^{2}}$}} & ventas anuales & \multirow[t]{2}{*}{$\$ 5,193.53$} \\
\hline feb-21 & 14 & 200 & 2800 & 196 & & & & & \\
\hline mar-21 & 15 & 200 & 3000 & 225 & \multirow{2}{*}{\multicolumn{2}{|c|}{$b=\frac{11220}{6936}$}} & \multirow[t]{2}{*}{1.61764706} & & \\
\hline$a b r-21$ & 16 & 200 & 3200 & 256 & & & & & \\
\hline may-21 & 17 & 200 & 3400 & 289 & & \multirow{2}{*}{\multicolumn{2}{|c|}{$\mathrm{a}=\frac{\sum y-b \sum x}{N}$}} & & \\
\hline Sumatoria & 153 & 3280 & 30180 & 1785 & & & & & \\
\hline $\mathbf{N}$ & 17 & & & & & 3032.5 & 178.382353 & & \\
\hline
\end{tabular}

Figura 6. Pronóstico de demanda Asogualel. Elaboración: Los autores.

Con base en los datos formulados y con la finalidad de analizar si la regresión es válida y se la puede utilizar para predecir las ventas, se procede a calcular el margen de error utilizando el método de error porcentual absoluto medio (PEMA) (ver figura 7), obteniendo un margen de error del $14.07 \%$, el mismo que debe ser considerado, en virtud de que, en los años 2019 al 2021 la producción fue irregular por la presencia de la pandemia de Covid-19. 


\begin{tabular}{|c|c|c|c|c|c|c|}
\hline periodo & yt & Pronóstico yt & et & abs (et) & et 2 & abs (et) /yt \\
\hline 1 & 180 & 208 & -27.50 & 27.50 & 756.25 & 0.15 \\
\hline 2 & 180 & 209 & -29.12 & 29.12 & 847.84 & 0.16 \\
\hline 3 & 180 & 211 & -30.74 & 30.74 & 944.66 & 0.17 \\
\hline 4 & 180 & 212 & -32.35 & 32.35 & 1046.71 & 0.18 \\
\hline 5 & 180 & 214 & -33.97 & 33.97 & 1154.00 & 0.19 \\
\hline 6 & 180 & 216 & -35.59 & 35.59 & 1266.52 & 0.20 \\
\hline 7 & 200 & 217 & -17.21 & 17.21 & 296.04 & 0.09 \\
\hline 8 & 200 & 219 & -18.82 & 18.82 & 354.33 & 0.09 \\
\hline 9 & 200 & 220 & -20.44 & 20.44 & 417.84 & 0.10 \\
\hline 10 & 200 & 222 & -22.06 & 22.06 & 486.59 & 0.11 \\
\hline 11 & 200 & 224 & -23.68 & 23.68 & 560.58 & 0.12 \\
\hline 12 & 200 & 225 & -25.29 & 25.29 & 639.79 & 0.13 \\
\hline & & & -316.76 & 316.76 & 8771.15 & 1.69 \\
\hline \multicolumn{2}{|r|r|r|r|}{} \\
\hline
\end{tabular}

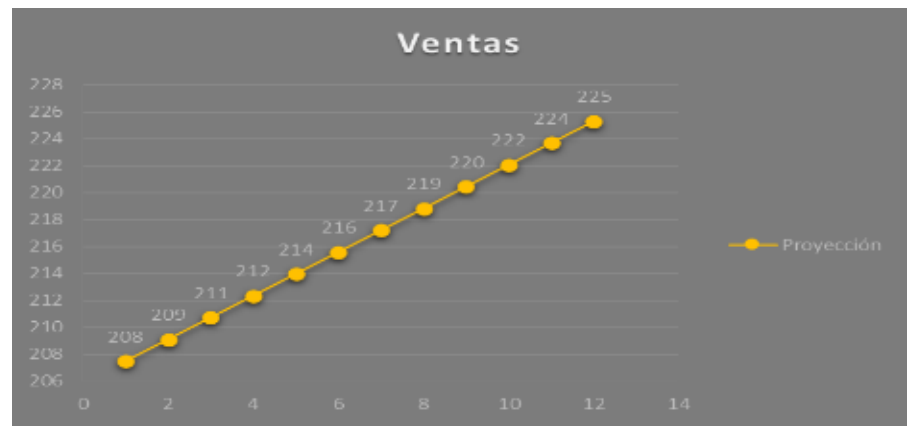

Figura 7. Margen de error pronóstico de ventas.

Elaboración: Los autores.

3. Planeación de producción: en la figura 8 se muestra el presupuesto de producción, el mismo que, guarda concordancia con el pronóstico de demanda y se ha preparado con los estándares de materiales y MO actuales, por tanto, debe ser observado por las unidades productivas y vigilado por el administrador, para de esta manera, evitar la formación inventarios finales debido a alta perecibilidad de los productos, así como, identificar el volumen necesario de los materiales necesarios para cada lote de producción. 


\section{CIENCIAMATRIA}

Revista Interdisciplinaria de Humanidades, Educación, Ciencia y Tecnología

Año VII. Vol. VII. N²2. Edición Especial II. 2021

Hecho el depósito de ley: pp201602FA4721

ISSN-L: 2542-3029; ISSN: 2610-802X

Universidad Nacional Experimental Francisco de Miranda (UNEFM). Santa Ana de Coro. Venezuela

Mónica Silvana Jiménez-Ren; Cecilia Ivonne Narváez-Zurita

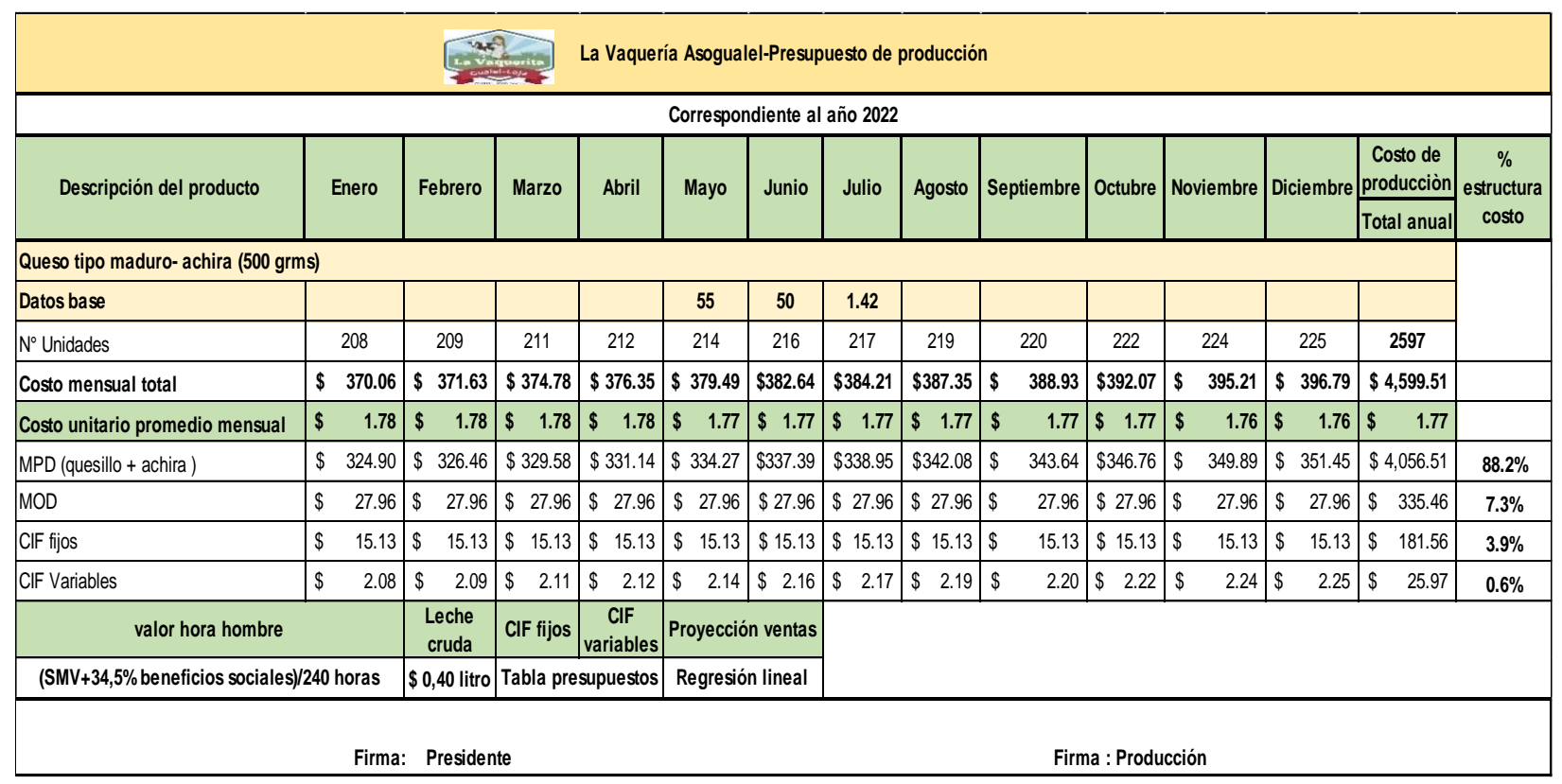

Figura 8. Presupuesto de producción.

Elaboración: Los autores.

4. Estructura de costos: en la actualidad, Asogualel produce bajo pedido de los clientes al no disponer de un punto de venta específico. En este sentido, se sugiere acumular los costos de producción utilizando el sistema por órdenes de trabajo.

a) Presupuesto de los CIF: cuando se utiliza el sistema por órdenes de trabajo, es conveniente trabajar con tasas predeterminadas de los CIF, a fin de, facilitar la inmediata liquidación o determinación de los costos de producción de cada orden. Para este efecto, se ha construido el siguiente presupuesto para el año 2022 (ver figura 9). 


\section{CIENCIAMATRIA}

Revista Interdisciplinaria de Humanidades, Educación, Ciencia y Tecnología

Año VII. Vol. VII. N². Edición Especial II. 2021

Hecho el depósito de ley: pp201602FA4721

ISSN-L: 2542-3029; ISSN: 2610-802X

Universidad Nacional Experimental Francisco de Miranda (UNEFM). Santa Ana de Coro. Venezuela

Mónica Silvana Jiménez-Ren; Cecilia Ivonne Narváez-Zurita

\begin{tabular}{|c|c|c|c|c|c|c|c|c|c|c|}
\hline \multicolumn{11}{|c|}{ gespand } \\
\hline \multicolumn{11}{|c|}{ Presupuesto para el año 2022} \\
\hline Descripción & \multicolumn{2}{|c|}{ Queso } & Yogur & $\begin{array}{l}\text { Dulce de } \\
\text { leche }\end{array}$ & \multicolumn{2}{|c|}{$\begin{array}{c}\text { Leche } \\
\text { pausterizada }\end{array}$} & \multicolumn{2}{|c|}{ Quesillo } & \multicolumn{2}{|c|}{ Producción Totla en gramos } \\
\hline Unidad de medida & \multicolumn{2}{|r|}{500 grms } & litro $=1000$ grms & 250 grms & \multicolumn{2}{|c|}{ litro $=1000$ grms } & \multicolumn{2}{|r|}{500 Grms } & & \\
\hline Cantidad & \multicolumn{2}{|c|}{208 unidades } & 96 litros & 67unidades & \multicolumn{2}{|c|}{832 litros } & \multicolumn{2}{|c|}{208 unidades } & \\
\hline Cantidad en gramos & \multicolumn{2}{|c|}{\begin{tabular}{|r|}
104000 \\
\end{tabular}} & 96000 & \begin{tabular}{r|}
16750 \\
00649
\end{tabular} & \multicolumn{2}{|r|}{832000} & \multicolumn{2}{|c|}{104000} & & \\
\hline CIF variables por unidad & $\$$ & \begin{tabular}{l|l}
0.0100 & $\$$ \\
\end{tabular} & 0.2104 & 0.0649 & $\$$ & 0.0224 & $\$$ & - & \multicolumn{2}{|r|}{1152750} \\
\hline Variables unitario lote & $\$$ & 2.08 & 20.20 & 4.35 & $\$$ & 18.64 & $\$$ & - & & \\
\hline \multicolumn{11}{|l|}{ MPI } \\
\hline Sal & $\$$ & 2.08 & & & & & & & & \\
\hline Funda plástica & & & & & $\$$ & 16.64 & & & & \\
\hline Envase & & & 19.20 & 3.35 & & & & & & \\
\hline Gas & & & 1.00 & 1.00 & $\$$ & 2.00 & & & & \\
\hline CIF fijos mensuales & \multicolumn{2}{|c|}{ Queso } & Yogur & $\begin{array}{c}\text { Dulce de } \\
\text { leche }\end{array}$ & pas & $\begin{array}{l}\text { Leche } \\
\text { Isterizada }\end{array}$ & & Zuesillo & Total mes & $\begin{array}{c}\text { Criterio de } \\
\text { distribución }\end{array}$ \\
\hline Carga fabril & $\$$ & \begin{tabular}{l|l}
15.13 & $\$$ \\
\end{tabular} & 14.41 & 10.08 & $\$$ & 69.82 & $\$$ & 10.97 & 120.42 & \\
\hline Depreciaciones (ver anexo) & $\$$ & \begin{tabular}{l|l}
4.17 & $\$$ \\
\end{tabular} & 4.00 & 5.17 & $\$$ & 8.33 & & & 21.67 & Asignación directa \\
\hline Arriendo de local & $\$$ & \begin{tabular}{l|l}
2.00 & $\$$ \\
\end{tabular} & 2.00 & 2.00 & $\$$ & 2.00 & $\$$ & 2.00 & 10.00 & Partes iguales \\
\hline Servicios básicos & $\$$ & \begin{tabular}{l|l}
1.00 & $\$$ \\
\end{tabular} & 1.00 & 1.00 & $\$$ & 1.00 & $\$$ & 1.00 & 5.00 & Partes iguales \\
\hline Otros no especificados & $\$$ & \begin{tabular}{l|l}
7.22 & $\$$ \\
\end{tabular} & 6.66 & 1.16 & $\$$ & 57.74 & $\$$ & 7.22 & 80.00 & gramos lote \\
\hline Guantes & $\$$ & \begin{tabular}{l|l}
0.50 & $\$$ \\
\end{tabular} & 0.50 & 0.50 & $\$$ & 0.50 & $\$$ & 0.50 & 2.50 & Partes iguales \\
\hline Gorros & $\$$ & \begin{tabular}{l|l}
0.25 & $\$$ \\
\end{tabular} & 0.25 & 0.25 & $\$$ & 0.25 & $\$$ & 0.25 & 1.25 & Partes iguales \\
\hline & & & Listado de ac & tivos fijos & & & & & & \\
\hline Descripción & & $\begin{array}{c}\text { Costo } \\
\text { hitórico }\end{array}$ & vida útil & $\begin{array}{c}\text { Depreciacic } \\
\text { anual }\end{array}$ & & \begin{tabular}{|c} 
Depreciac \\
mes \\
\end{tabular} & ión & usu & uario & \\
\hline Molino electrico & $\$$ & 250.00 & 5 & & .00 & $\$$ & .17 & Queso & & \\
\hline Cocina semi industrial & $\$$ & 60.00 & 5 & & 2.00 & $\$$ & .00 & Yogur & & \\
\hline 2 Cilintro de gas & $\$$ & 120.00 & 5 & & 4.00 & $\$$ & .00 & $\begin{array}{l}\text { Yogur y c } \\
\text { leche }\end{array}$ & dulce de & \\
\hline Licuadora & $\$$ & 120.00 & 5 & & 4.00 & $\$$ & 2.00 & Yogur & & \\
\hline Molino electrico & $\$$ & 250.00 & 5 & & .00 & $\$$ & .17 & Dulce de & leche & \\
\hline otros (horno ollas filtros etc) & $\$$ & 500.00 & 5 & 100 & 0.00 & $\$$ & 33 & Leche pa & asteurizada & \\
\hline TOTAL & $\$$ & $1,300.00$ & & 160 & 0.00 & $\$$ & .67 & & & \\
\hline
\end{tabular}

Figura 9. Presupuesto de CIF 2022

Elaboración: Los autores.

Orden de trabajo o de producción: permite planificar el proceso de producción, en virtud de que, contiene toda la información pertinente para ejecutar la producción, esto es, fechas programadas de inicio y culminación de la producción para satisfacer a tiempo la demanda, materiales y recurso necesarios para ejecutar la orden (ver figura 10) 


\section{CIENCIAMATRIA}

Revista Interdisciplinaria de Humanidades, Educación, Ciencia y Tecnología

Año VII. Vol. VII. N². Edición Especial II. 2021

Hecho el depósito de ley: pp201602FA4721

ISSN-L: 2542-3029; ISSN: 2610-802X

Universidad Nacional Experimental Francisco de Miranda (UNEFM). Santa Ana de Coro. Venezuela

Mónica Silvana Jiménez-Ren; Cecilia Ivonne Narváez-Zurita

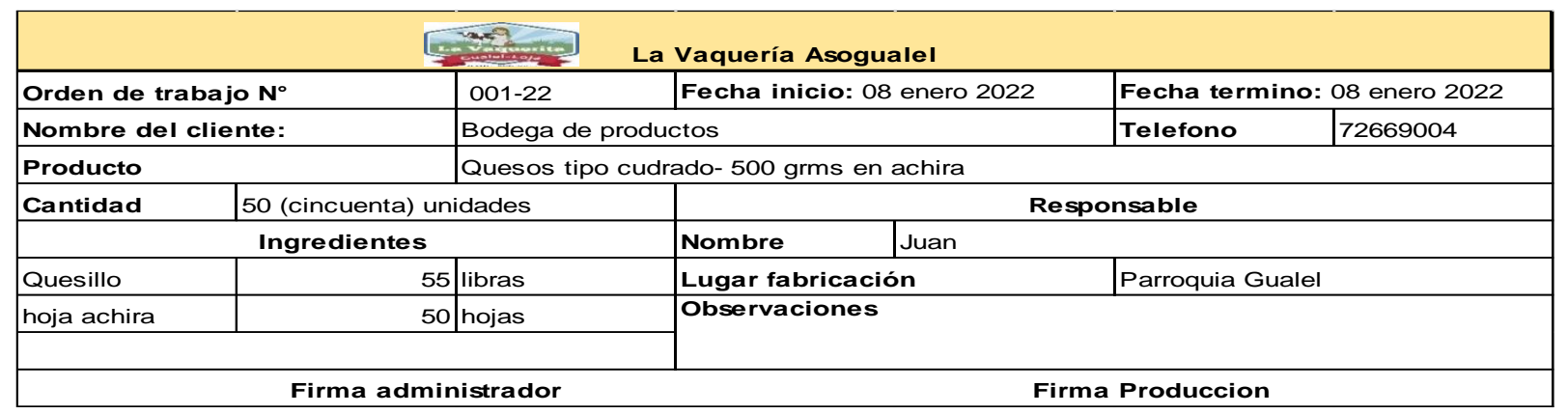

Figura 10. Orden de trabajo.

Elaboración: Los autores.

b) Requisición y despacho de materiales: emitida la orden de trabajo, de inmediato se envía al taller las notas de despacho de materiales (ver figura 11).

\begin{tabular}{|c|c|c|c|c|c|c|c|}
\hline & 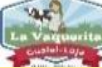 & \multicolumn{2}{|c|}{ La Vaqueria Asogualel } & & & & \\
\hline \multicolumn{8}{|c|}{ Nota de despacho de materiales № 100} \\
\hline \multicolumn{8}{|c|}{ Orden de producción № $01-22$} \\
\hline \multicolumn{2}{|c|}{ Responsable................. } & Centro de costo & \multicolumn{5}{|c|}{ 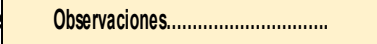 } \\
\hline \multirow{2}{*}{ Cantidad } & \multirow{2}{*}{$\begin{array}{l}\text { unidad de } \\
\text { medida }\end{array}$} & \multirow{2}{*}{ Descripción } & \multirow{2}{*}{ código } & \multicolumn{4}{|c|}{ costo } \\
\hline & & & & & & & tal \\
\hline \multicolumn{2}{|c|}{55 |ibras } & quesillo tresco & 1.100 .001 & $\$$ & 1.40 & $\$$ & 77.00 \\
\hline & & Suman \$ & & & & & \\
\hline \multicolumn{3}{|c|}{ Recibe: } & \multicolumn{5}{|c|}{ Entrega: } \\
\hline \multicolumn{3}{|c|}{ Taller industrial } & \multicolumn{5}{|c|}{ Bodeguero } \\
\hline
\end{tabular}

\begin{tabular}{|c|c|c|c|c|c|}
\hline & $\begin{array}{l}42 \\
4 \\
2+2\end{array}$ & \multicolumn{4}{|c|}{ La Vaquería Asogualel } \\
\hline \multicolumn{6}{|c|}{ Nota de despacho de materiales № 101} \\
\hline \multicolumn{6}{|c|}{ Orden de producción № $01-22$} \\
\hline \multicolumn{2}{|c|}{ Responsable.................. } & Centro de costo & \multicolumn{3}{|c|}{ 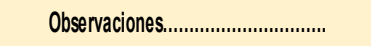 } \\
\hline \multirow{2}{*}{ Cantidad } & \multirow{2}{*}{$\begin{array}{l}\text { unidad de } \\
\text { medida }\end{array}$} & \multirow{2}{*}{ Descripción } & \multirow{2}{*}{ código } & \multicolumn{2}{|c|}{ costo } \\
\hline & & & & unitario & total \\
\hline \multicolumn{2}{|c|}{ 50 unidad } & hoja de achira & 1.100 .002 & 0.02 & 1.00 \\
\hline & & Suman \$ & & & \\
\hline \multicolumn{3}{|c|}{ Recibe: } & \multicolumn{3}{|c|}{ Entrega: } \\
\hline \multicolumn{3}{|c|}{ Taller industrial } & \multicolumn{3}{|c|}{ Bodeguero } \\
\hline
\end{tabular}

Figura 11. Notas de despacho $\mathrm{N}^{\circ} 100$ y 101. Elaboración: Los autores. 
c) Asignación MO: todos los componentes de la remuneración se integran y de ahí se dividen para el número de horas-mes que deben asistir los obreros. De aquí se obtiene el valor de cada hora de trabajo de $\$ 2.24$ (ver figura 12).

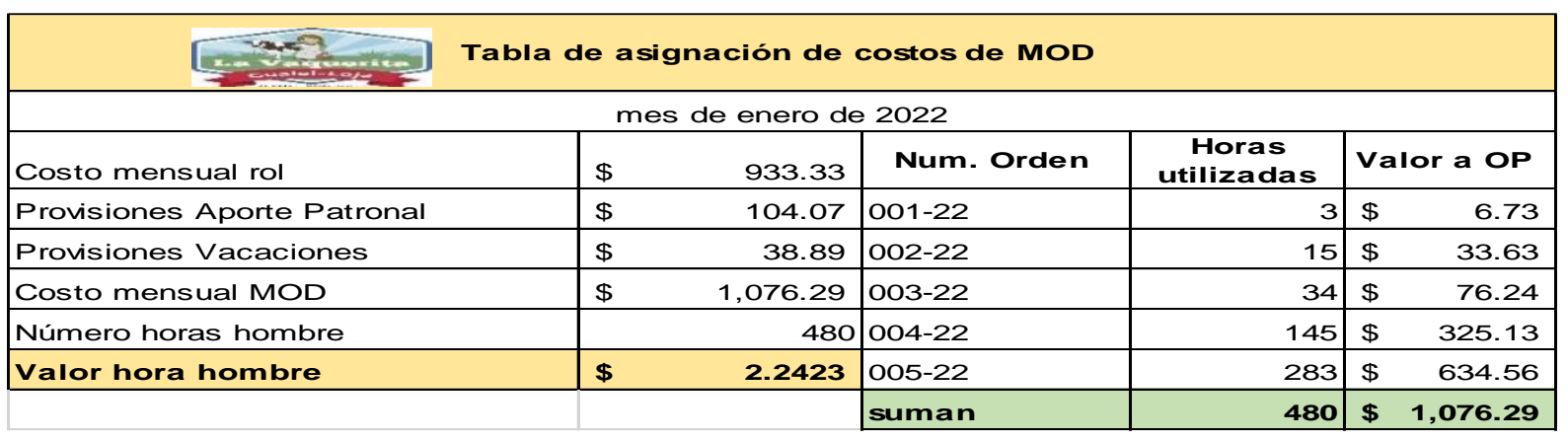

Figura 12. Asignación de costos MOD. Elaboración: Los autores.

d) Hoja de costos (libro auxiliar): este documento facilita el control de los costos de producción (inversión). En efecto, en la figura 12 se evidencia la acumulación de cada uno de los elementos del costo incurrido en la orden de trabajo Nro. 001-22. Al finalizar la semana, se computa las horas de trabajo de los socios-obreros que laboraron en esta orden y se hace el cargo de los CIF tomando los datos presupuestados con anticipación (ver figura 13). 
Revista Interdisciplinaria de Humanidades, Educación, Ciencia y Tecnología

Año VII. Vol. VII. N². Edición Especial II. 2021

Hecho el depósito de ley: pp201602FA4721

ISSN-L: 2542-3029; ISSN: 2610-802X

Universidad Nacional Experimental Francisco de Miranda (UNEFM). Santa Ana de Coro. Venezuela

Mónica Silvana Jiménez-Ren; Cecilia Ivonne Narváez-Zurita

\begin{tabular}{|c|c|c|c|c|c|c|c|}
\hline \multicolumn{8}{|c|}{ La Vaquería Asogualel } \\
\hline \multicolumn{8}{|c|}{ Hoja de costos } \\
\hline \multicolumn{8}{|c|}{ Orden de producción $\mathrm{N}^{\circ}$ 01-22 } \\
\hline \multicolumn{3}{|c|}{ Cliente: Bodega de productos } & $: 8$ enero 2022 & Fecha terminac & ción: 8 enero 2022 & & \\
\hline \multicolumn{8}{|c|}{ Producto: Queso 500 grms cuadrado Especificación: Envuelto en hoja achira Cantidad: 50 unidades } \\
\hline \multicolumn{3}{|c|}{ MPD } & \multicolumn{5}{|c|}{ MOD } \\
\hline Fecha & Docum. № & Importe & Fecha & № horas & Val. hora & & \\
\hline 8 enero & Nota egreso 100 & \begin{tabular}{l|l}
77.10 & $\varepsilon$ \\
\end{tabular} & 8 enero & 3.00 & 2.2423 & $\$$ & 6.727 \\
\hline 8 enero & \begin{tabular}{|l|} 
Nota egreso 101 \\
\end{tabular} & $\$ 1.00$ & & & & & \\
\hline \multicolumn{2}{|c|}{ Suma $\$$} & $\$ 78.10$ & \multicolumn{3}{|c|}{ Suma $\$$} & $\$$ & 6.727 \\
\hline \multicolumn{3}{|r|}{ Resumen } & \multicolumn{5}{|c|}{ CIF } \\
\hline \multicolumn{2}{|c|}{ Concepto } & Costo Total & Costo Unitario & Fecha & $\begin{array}{l}\text { Tasa Predet } x \\
\text { unidad }\end{array}$ & \multicolumn{2}{|c|}{ Importe } \\
\hline \multicolumn{2}{|l|}{ MPD } & $\$ 78.10$ & 1.5620 & 8 enero (fijo) & 0.0728 & $\$$ & 3.64 \\
\hline \multicolumn{2}{|l|}{ MOD } & 6.727 & 0.1345 & 8 enero (varia) & 0.0100000 & & 0.50 \\
\hline \multicolumn{2}{|l|}{ Costo primo } & $\$ 84.83$ & 1.6965 & & & & \\
\hline \multicolumn{2}{|l|}{ CIF } & 4.14 & 0.0828 & & & & \\
\hline \multicolumn{2}{|c|}{ Costo de producción } & $\$ 88.97$ & 1.7793 & & Suma \$ & $\$$ & 4.14 \\
\hline
\end{tabular}

Figura 13. Hoja de costos.

Elaboración: Los autores.

Finalizada la producción el sábado 8 enero 2022, se obtiene la cantidad deseada de 50 quesos de 500 gramos en el formato cuadrado envuelto en hoja de achira, que se destina a la bodega para la venta a intermediarios o clientes. Donde el costo unitario de producción asciende a $\$ 1.77$ y se estima vender a $\$ 2.00$ cada unidad.

e) Registro contable: A manera de ejemplo, se proponen los registros en el diario de la orden de producción № 01-22, desde la inversión, liquidación de la orden e ingreso a bodega de los productos y culmina con la venta inmediata de toda la producción (ver figura 14). 


\section{CIENCIAMATRIA}

Revista Interdisciplinaria de Humanidades, Educación, Ciencia y Tecnología

Año VII. Vol. VII. N². Edición Especial II. 2021

Hecho el depósito de ley: pp201602FA4721

ISSN-L: 2542-3029; ISSN: 2610-802X

Universidad Nacional Experimental Francisco de Miranda (UNEFM). Santa Ana de Coro. Venezuela

Mónica Silvana Jiménez-Ren; Cecilia Ivonne Narváez-Zurita

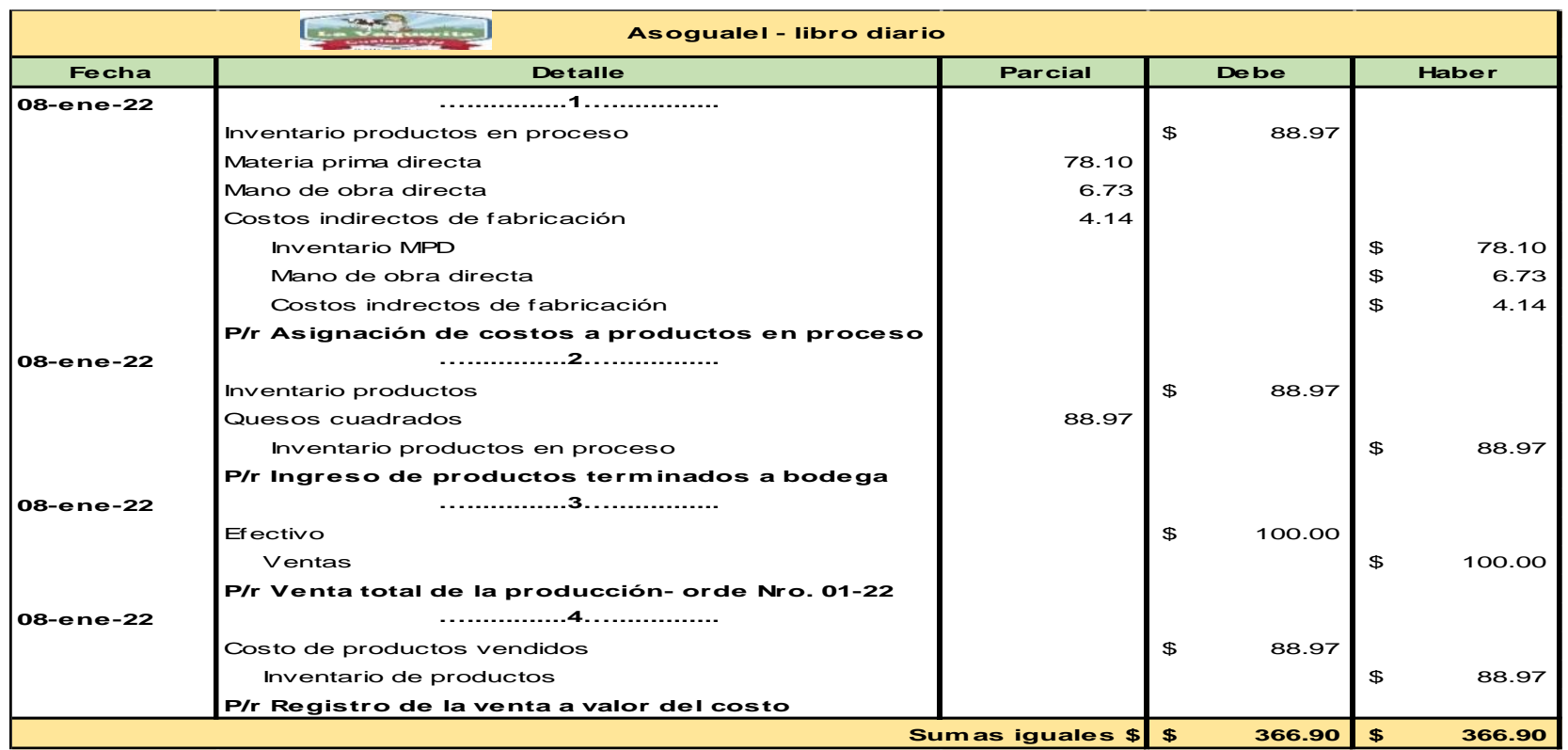

Figura 14: Libro diario con asientos tipo.

Elaboración: Los autores.

5. Punto de equilibrio: el cálculo del punto de equilibrio, parte de la identificación del precio de venta y del costo variable unitario, de manera que, la diferencia determina el margen de contribución unitario. Como complemento, se deben establecer los gastos fijos que se consumen en las funciones administrativas y comerciales (ver figura 15). 


\begin{tabular}{|l|cc|}
\hline \multicolumn{3}{|c|}{ Comerciales } \\
\hline Remuneraciones & $\$$ & - \\
\hline Servicios básicos & $\$$ & 150.00 \\
\hline Promoción & $\$$ & 300.00 \\
\hline Otros & $\$$ & 100.00 \\
\hline Suman \$ & $\$$ & $\mathbf{5 5 0 . 0 0}$ \\
\hline
\end{tabular}

\begin{tabular}{|l|lr|}
\hline \multicolumn{3}{|c|}{ Administrativos } \\
\hline Remuneraciones & $\$$ & $3,600.00$ \\
\hline Servicios básicos & $\$$ & 40.00 \\
\hline depreciaciones & $\$$ & 90.00 \\
\hline & & \\
\hline Suman \$ & $\$$ & $\mathbf{3 , 7 3 0 . 0 0}$ \\
\hline
\end{tabular}

Figura 15. Gastos comerciales y administrativos. Elaboración: Los autores.

Con estos supuestos se procede a calcular y mostrar el punto de equilibrio de Asogualel para el año 2022, exclusivamente del queso que es el producto estrella (ver figura 16).

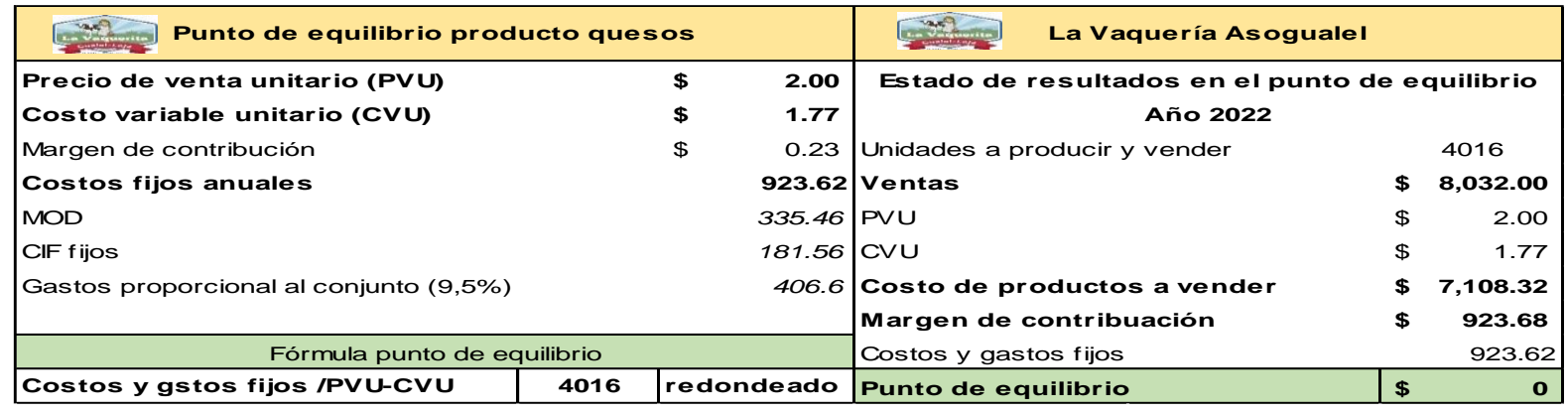

Figura 16. Punto de equilibrio. Elaboración: Los autores.

De los resultados expresados en la tabla 16 se determina que el punto de equilibrio es de 4016 unidades al año, lo que implica una venta de 11 quesos al día. Nótese que en la tabla 6 del pronóstico de demanda se tiene una proyección aproximada de 2597 unidades al año, por tanto, si se mantiene el mismo ritmo de producción como el que se maneja en la actualidad no se alcanza este punto de equilibrio, por el contrario, se obtiene una pérdida en razón de que los gastos fijos administrativos y comerciales son mínimos y no se pueden disminuir. No obstante, por citar un ejemplo, para que esta organización obtenga una utilidad anual de $\$ 500,00$ que es razonable, tendría que producir y vender 
6190 quesos en un promedio de 17 unidades diarias, de lo contrario la utilidad esperada no se podrá alcanzar.

\section{CONCLUSIONES}

Toda empresa sin importar su tamaño debe tener un control apropiado de sus recursos, que garanticen su sostenibilidad, estabilidad y continuidad. De manera que, a través de la contabilidad y sus registros se obtenga información de las transacciones comerciales y financieras que de una $u$ otra forma afectan a los citados recursos, por tanto, su importancia no solo radica en la función tributaria, sino que, permite medir de forma objetiva el desempeño del negocio, hacer proyecciones que guían el camino a seguir y tomar decisiones inteligentes y acertadas a partir de los datos acumulados y registrados del pasado.

Las organizaciones que aspiran a ser competitivas deben gestionar de forma adecuada los costos, es decir, manejar de manera eficiente, económica y eficaz sus recursos, de ahí la necesidad de contar con un sistema de costos que incluya técnicas de control y gestión que faciliten la optimización de recursos e incentiven el trabajo eficiente y de calidad.

Una de las debilidades más significativas de Asogualel radica en la carencia de un sistema contable que suministre información confiable, oportuna y relevante que se obtiene de la contabilidad bajo normas internacionales. Tal es así que, se evidencian problemas económicos, de producción, ventas no planificadas, costos y gastos no controlados y obviamente, los resultados económicos esperados como utilidad no es posible alcanzar. Por tanto, es importante mantener un registro contable actualizado y razonable de los hechos económicos generados, que sirva de base para la toma de decisiones estratégicas, a partir de las cuales esta microempresa puede crecer de manera ordenada y sostenida en el tiempo. 
Otro hallazgo significativo se evidencia en el proceso productivo, el mismo que, no es planificado ni controlado. Con base en la problemática expuesta, se sugiere un modelo de planeación del costo de producción, que permita conocer lo que se debe producir, cuándo (periodos) producir y cuánto (cantidad) producir. Esta herramienta, complementada con una estructura de costos adecuada, permitirá que Asogualel logre optimizar los recursos y sacar el máximo rendimiento, ofreciendo sus productos en la cantidad y tiempo adecuados.

\section{REFERENCIAS}

Albán-Vallejo, V., Betancourt-Soto, V., \& Morales-Merchán, N. (2015). El costo de producción y la fijación de precios en las microempresas [The cost of production and pricing in micro-enterprises]. Eumednet, 1-12. Obtenido de https://n9.cl/f8xp

Alcívar-Cedeño, F. (2017). Modelo de costeo para producción de quesos en microempresas comunitarias Salinas de Bolívar [Cost model for cheese production in Salinas de Bolívar community micro-enterprises]. Guayaquil: Universidad de Guayaquil . Obtenido de https://n9.cl/0uz4r

Alvarado-Martínez, T. (2001). Metodología para elaborar un plan estratégico y rediseño organizacional de una unidad de producción agropecuaria [Methodology for developing a strategic plan and organizational redesign of an agricultural production unit]. Revista Mexicana de Agronegocios, 284-292. Obtenido de https://n9.cl/hbajr

Álvarez-Morales, E., \& Lazo-Planas, L. (2017). El sistema de costeo por actividades en las pymes con responsabilidad social empresarial [Activity Based Costing System in Small and Medium Enterprises with Corporate Social Responsibility]. Cofín Habana, 46-56. Obtenido de https://n9.cl/sy3di

Arellano-Cepeda, O., Quispe-Fernández, G., Ayaviri-Nina, D., \& Escobar-Mamani, F. (2017). Estudio de la Aplicación del Método de Costos ABC en las Mypes del Ecuador [Study of the Application Of The ABC Cost Method in the Mypes Of Ecuador]. Revista de Investigaciones Altoandinas, 33-45. doi: http://dx.doi.org/10.18271/ria.2016.253 
Artieda, C. (2015). Análisis de los sistemas de costos como herramientas estratégicas de gestión en las pequeñas y medianas empresas (PYMES) [Analysis of cost systems as strategic management tools in small and medium-sized enterprises (SMEs)]. Revista Publicando, 90-113. Obtenido de https://n9.cl/rihb

Barriga-Pizarro, M., Asunción-Parralees, R., Sánchez-Segarra, J., \& Balseca-Córdova, M. (2020). La importancia de la contabilidad de costos en el control administrativo de los emprendedores [The importance of cost accounting in the administrative control of entrepreneurs]. Revista científica Aristas, 48-62. Obtenido de https://n9.cl/j28kwz

Bazan-Liñan, L., \& Berrospi-Valverde, S. (2018). Costos de producción y utilidad. [Production and utility costs]. Trujillo: Repositorio de la Universidad Privada del Norte. Obtenido de https://n9.cl/8c9sp

Carpio-Valencia, F. (2019). Costos estándar para optimizar la rentabilidad de los productores artesanales de queso: caso PROLAC AYMARA [Standard costs to optimize the profitability of handmade producers of cheese: PROLAC AYMARA case]. Dyna, 262-269. doi:http://doi.org/10.15446/dyna.v86n210.77995

Chávez-Jaramillo, M., Narváez-Zurita, C., Ormaza-Andrade, J., \& Erazo-Álvarez, J. (2019). Gestión de costos ABC/ABM en la industria avícola del Ecuador. Caso de aplicación: Franksur Industrial Avícola Cía. Ltda [ ABC/ABM cost management in Ecuador's poultry industry. Application case: Franksur Industrial Poultry Cía. Ltda]. Visionario Digital, 284-308. doi:https://doi.org/10.33262/visionariodigital.v3i2.1.556

Condori-Muñiz, Y., \& Maraza-Humpire, R. (2017). Los costos de producción y su relación con el precio de venta en la empresa exportadora orgánica SAC Distrito de Santiago de Surco [Production costs and their relationship to the sale price in the organic exporting company SAC Distrito de Santiago de Sur]. Lima: Universidad Autónoma del Perú. Obtenido de https://n9.cl/kx5uu

Cortés-Cortés, J. (01 de 01 de 2015). Heurísticas y toma de decisiones gerenciales individuales en pymes de Bogotá [Heuristics and individual management decisionmaking in Bogota SMEs]. Bogotá: Universidad Nacional de Colombia. Obtenido de https://n9.cl/3noiw 
Franklin-Fincowsky, E. (2011). Toma de decisiones empresariales. Reseña de "Comportamiento organizacional, enfoque para América Latina" [Business decision-making. Review of "Organizational Behavior, Focus for Latin America"]. Contabilidad y Negocios, 113-120. Obtenido de https://n9.cl/2u0x

Garrison, R., Noreen, E., \& Brewer, P. (2007). Contabilidad administrativa [Administrative accounting] (11va ed.). México: McGraw-Hill Interamericana Editores S.A.

Hansen, D., \& Mowen, M. (2007). Administración de costos. Contabilidad y control [Cost management. Accounting and control] (5ta ed.). Santa Fe: Cengage Learning Editores, S.A.

Lazo-Palacios, M. (2013). Contabilidad de Costos I [Cost Accounting I] (1era ed.). Lima: Centro de Producción de Materiales Académicos (CEPMA).

Mendoza-Torres, C., \& Campos-Mendoza, M. (2021). Administración de costos en las pymes de transformación en la región Laja Bajío de México [Cost management in processing SMEs in Mexico's Laja Bajío region]. Signos-Investigación en Sistemas de Gestión, 45-59. doi:https://doi.org/10.15332/24631140.6340

Morocho-Malla, L., Narváez-Zurita, C., \& Erazo-Álvarez, J. (2019). La determinación de costos a través de la metodología abc/abm como opción estratégica en la industria de servicios portuarios [The determination of costs through the abc / abm methodology as a strategic option in the port services industry]. Cienciamatria, 418-447. doi: https://doi.org/10.35381/cm.v5i1.273

Pazquel-Barroso, Y. (2015). Implementación de un sistema de costos por procesos de los derivados de la leche y su incidencia en la rentabilidad [Implementation of a process cost system for milk derivatives and its impact on the profitability of the milk]. La Maná: Repositorio digital Unidad Académica de Ciencias Administrativas y Humanísticas. UTC. Obtenido de https://n9.cl/rnzg

Pilay-Toala, F. (2015). Microeconomía en la toma de decisiones gerenciales [Microeconomics in management decision-making]. ECA Sinergia, 18-28. Obtenido de https://n9.cl/rfxzh 
Ramírez-Molinares, C., García-Barbosa, M., \& Panoja-Algarín, C. (2010). Fundamentos y técnicas de costos [Basics and cost techniques]. Cartagena de las Indias: Editorial Universidad Libre, sede Cartagena. Obtenido de https://n9.cl/9xvx

Ramírez-Padilla, D. (2008). Contabilidad administrativa [Administrative accounting] (8va ed.). México: McGraw-Hill Interamericana.

Ramos-Farroñan, E., Mortocarrero-Medina, Y., \& Portocarrero-Medina, Y. (2020). El sistema de Costos $A B C$ como estrategia para la toma de decisiones empresarial [The ABC cost system as a strategy for the decision making of a company]. Universidad y Sociedad, 178-183. Obtenido de https://n9.cl/zfvc7

Rodríguez-Espinosa, H., Ramírez-Gómez, C., \& Restrepo-Betancur, L. (2017). Factores determinantes de la sostenibilidad de las agroempresas asociativas rurales [Determinants of the sustainability of rural associative agribusinesses]. Revista de economía e Sociologia Rural, 107-122. doi:https://doi.org/10.1590/1234$\underline{56781806-94790560107}$

Sarmiento-Regalado, R. (2005). Contabilidad de costos [Cost accounting] (1era ed.). Quito: Cámara Ecuatoriana del Libro-Núcleo de Pichincha.

Uribe-Marín, R. (2011). Costos para la toma de decisiones [Decision-making costs] (1era ed.). Bogotá: McGraw-Hill Interamericana S.A.

Vallejos-Orbe, H., \& Chiliquinga-Jaramillo, M. (2017). Costos modalidad órdenes de producción [Costs modality production orders]. Ibararra: Universidad Técnica del Norte (UTN). Obtenido de https://n9.cl/n2d8

Vera-Franco, P., Espinoza-Cume, A., \& López-Coloma, R. (2016). La importancia de la contabilidad en las empresas [The importance of accounting in companies]. Contribuciones a la Economía , 1-12. Obtenido de https://n9.cl/z3bxt

Vinza-Romero, S. (2012). Propuesta para la aplicación del método ABC de la industria gráficas olmedo [Proposal for the application of the $A B C$ method of the industry olmedo graphics]. Quito: Universidad Central del Ecuador (UCE). Obtenido de https://n9.cl/k1fzc 


\section{CIENCIAMATRIA}

Revista Interdisciplinaria de Humanidades, Educación, Ciencia y Tecnología

Año VII. Vol. VII. N². Edición Especial II. 2021

Hecho el depósito de ley: pp201602FA4721

ISSN-L: 2542-3029; ISSN: 2610-802X

Universidad Nacional Experimental Francisco de Miranda (UNEFM). Santa Ana de Coro. Venezuela

Mónica Silvana Jiménez-Ren; Cecilia Ivonne Narváez-Zurita

Zapata-Sánchez, P. (2019). Contabilidad de Costos [Cost Accounting] (3era ed.). Bogotá: Alfaomega Colombiana S.A.

(C2021 por los autores. Este artículo es de acceso abierto y distribuido según los términos y condiciones de la licencia Creative Commons Atribución-NoComercial-Compartirlgual 4.0 Internacional (CC BY-NC-SA 4.0)

(https://creativecommons.org/licenses/by-nc-sa/4.0/). 